\title{
CHAIN CONDITIONS ON ESSENTIAL SUBMODULES
}

\author{
BARBARA L. OSOFSKY
}

(Communicated by Maurice Auslander)

\begin{abstract}
For $\aleph$ an infinite cardinal and $M$ a unital right module over a ring $R$ with 1 or an object in an $\mathscr{A B B} 5$ category, we show that every well ordered ascending (respectively descending) chain of essential submodules of $M$ has cardinality less than $\mathcal{N}$ if and only if every well ordered ascending (respectively descending) chain of submodules of $M / \operatorname{socle}(M)$ has cardinality less than $\aleph$. We use this to show that a $C S$ module with an $\aleph$-chain condition on essential submodules is a direct sum of a module with that same chain condition on all submodules plus a semisimple module. Thus a $C S$ module with fewer than $\aleph$ generators has an $\aleph$-chain condition on essential submodules if and only if it has that same $\aleph$-chain condition on all submodules. As an application in the case of an $\aleph_{0}$-chain condition, we describe the endomorphism ring of a continuous module with acc on essential submodules.
\end{abstract}

Chain conditions on a module (or object in an $\mathscr{A B S} 5$ category) appear in many contexts. We say that a partially ordered set $\mathscr{X}$ has the ascending (respectively descending) $\aleph$-chain condition iff for every ordinal $\kappa$ such that there is a chain $\left\{N_{\alpha} \mid \alpha<\kappa\right\}$ of subsets of $\mathscr{Z}$ with $N_{\beta}<N_{\alpha}$ (respectively $N_{\beta}>N_{\alpha}$ ) for all $\beta<\alpha \in \kappa$, we have $|\kappa|<\aleph$. Thus the usual acc and dcc are $\aleph_{0}$-chain conditions. Bass has shown ([3]) that any commutative Noetherian ring has the descending $\aleph_{1}$-chain condition on ideals. In contrast, Jategaonkar has shown ([7]) that right principal ideal domains can have descending chains of two sided ideals of any preassigned cardinality. Lawrence has shown ([8]) that a right self injective ring $R$ of cardinality $\aleph$ has the ascending $\aleph$-chain condition on annihilator right ideals. The Hopkins-Levitski theorem says that a ring with the descending $\aleph_{0}$-chain condition on right ideals has the ascending $\aleph_{0}$-chain condition on right ideals. There are valuation domain examples where the descending $\aleph_{1}$-chain condition does not imply the ascending $\aleph_{1}$-chain condition. Several authors have looked at the usual $\left(\aleph_{0^{-}}\right)$chain conditions on the subset of all essential submodules of a module and have obtained or used an $\aleph_{0}$ version of some of our theorems in the special case of ordinary acc and/or dcc. We mention, for example $[1,4,5,6]$. In this note we show that, for any infinite cardinal $\aleph$, an $\aleph$-chain condition on essential submodules of a module $M$ is very close to that $\aleph$-chain condition on all submodules. Specifically, $M$ has an

Received by the editors July $9,1990$.

1980 Mathematics Subject Classification (1985 Revision). Primary 16A53, 16A64; Secondary $16 \mathrm{~A} 65$.

Key words and phrases. Chain conditions, essential submodules. 
$\aleph$-chain condition on all submodules if and only if $M$ has that $\aleph$-chain condition on essential submodules and on sets of independent submodules. Also, $M$ has an $\aleph$-chain condition on essential submodules if and only if $M / \operatorname{socle}(M)$ has that $\aleph$-chain condition on all submodules. We apply the results to $C S$ modules, that is, modules for which any submodule is essential in a direct summand. We show that any such module with an $\aleph$-chain condition on essentials is a direct sum of a module with the $\aleph$-chain condition on all submodules and a semisimple module. In the case of the ascending $\aleph_{0}$-chain condition on essential submodules of a continuous module $M$ this enables us to describe the endomorphism ring of $M$.

$M$ will denote a fixed $R$-module or object in an $\mathscr{A} \mathscr{B} 5$ category, and $\aleph$ an infinite cardinal. For any set $\mathscr{X},|\mathscr{X}|$ will denote the cardinality of $\mathscr{X}$. $N \subseteq M$ will mean $N$ is a submodule of $M . N$ is an essential submodule of $M$, that is $N$ has nonzero intersection with each nonzero $L \subseteq M$, will be denoted $N \subseteq$ ' $M$. For $N \subseteq M$, a complement of $N$ is a submodule of $M$ maximal in $\{L \subseteq M \mid L \cap N=0\}$. A module is $C S$ if and only if every complement submodule is a direct summand. A family of submodules $\left\{N_{i} \mid i \in \mathscr{I}\right\}$ is called independent if the sum $\sum_{i \in \mathcal{I}} N_{i}$ is direct and the $N_{i}$ are all nonzero. Proofs are phrased in terms of (right) modules, but they are valid in an arbitrary $\mathscr{A B} 5$ category. For example, a categorical definition of $\aleph$-generated can be found in [10]. Reference [2] is a good source of background material. Several well known or easily established results are included as lemmas for convenience in referencing, sometimes with a brief sketch of a proof.

Lemma 1. If $M$ has an $\aleph$-chain condition on essential submodules, then so does every submodule and every quotient module of $M$.

Sketch of proof. Let $K \subseteq M$, and let $L$ be a complement of $K$ in $M$. Then adding $L$ to each member of a chain of submodules essential in $K$ gives a chain of submodules essential in $M$, so submodules inherit chain conditions on essentials. The inverse image of an essential submodule of $M / K$ is essential in $M$, so quotients also inherit chain conditions on essentials.

Theorem 1. Let $M$ be a module and $\aleph$ an infinite cardinal such that

(1) Any independent family of submodules of $M$ has fewer than $\aleph$ members.

(2) The set of all essential submodules of $M$ has the ascending (respectively descending) $\aleph$-chain condition.

Then the set of all submodules of $M$ has the ascending (respectively descending) $\aleph$-chain condition. Conversely, an $\aleph$-chain condition on all submodules of $M$ implies (1) and the same chain condition in (2).

Proof. The proof will be divided into three parts. To avoid interrupting the flow of ideas with messy details, only the first will be given here. It handles the case when $\aleph$ is a regular cardinal. This portion of the proof is straightforward and gives insight into why one would expect the theorem to be true. The proofs for the ascending and descending $\aleph$-chain condition when $\aleph$ is not regular are deferred to an appendix.

Part 1. $\aleph$ regular. Let $\left\{N_{\alpha} \mid \alpha<\kappa\right\}$ be an ascending (respectively descending) chain of distinct submodules of $M$. Define an equivalence relation $\sim$ on $\kappa$ by $\alpha \sim \beta$ iff $N_{\alpha} \subseteq^{\prime} N_{\beta}$ or $N_{\beta} \subseteq^{\prime} N_{\alpha}$. The set $\left\{N_{\alpha}\right\}$ corresponding to members of an equivalence class is an ascending (respectively descending) chain 
of submodules essential in the union of the set. Thus by Lemma 1, there are fewer than $\aleph$ members in each equivalence class. Also, each class has a smallest member $\bar{\alpha}$. The set $\mathscr{C}_{\sim}=\{\sim$-class representatives $\bar{\alpha}\}$ is a subchain of $\kappa$ and so well ordered. Let $\overline{\alpha^{+}}$denote the successor of $\bar{\alpha}$ in this subchain. For each $\overline{\alpha^{+}} \in \mathscr{C}_{\sim}$, the smaller of $\left\{N_{\bar{\alpha}}, N_{\overline{\alpha^{+}}}\right\}$has a nonzero complement $L_{\bar{\alpha}}$ in the larger. These $L_{\bar{\alpha}}$ are clearly independent and in one-to-one correspondence with the set of successors in $\mathscr{C}_{\sim}$. If $\mathscr{C}_{\sim}$ is infinite it has the same cardinality as the set of successors in it. Thus there are fewer than $\aleph$ equivalence classes by (1). If $\aleph$ is regular, then $\kappa$, being a union of less than $\aleph$ classes of less than $\aleph$ elements, must have $|\kappa|<\aleph$.

The converse of the theorem is clear.

Lemma 2. A module $N$ is semisimple $\Longleftrightarrow N$ has no proper essential submodules.

Sketch of proof. If $N$ is semisimple, then every submodule is a direct summand of $N$ and so not essential unless equal to $N$. Conversely, any $K \subseteq N$ has a complement $L \subseteq N$. Then $K \oplus L \subseteq^{\prime} N$, so if $N$ has no proper essential submodules, $K$ is a direct summand of $N$.

Theorem 2. Let $M$ be a module with either the ascending or descending $\aleph$-chain condition on essential submodules. Then every direct sum $\oplus_{\alpha \in \kappa} E_{\alpha} \subseteq M$, where each $E_{\alpha}$ is not semisimple, has fewer than $\aleph E_{\alpha}$.

Proof. Let $\oplus_{\alpha \in \kappa} E_{\alpha}$ be an infinite direct sum of nonsemisimple submodules of $M$. By adding a complement of $\oplus_{\alpha \in \kappa} E_{\alpha}$ to one of the $E_{\alpha}$ if necessary, we may assume that $\oplus_{\alpha \in \kappa} E_{\alpha}$ is essential in $M$. Without loss of generality we also may assume that $\kappa$ is an ordinal.

For each $\alpha \in \kappa$, select a proper essential submodule of $E_{\alpha}$ and denote it by $N_{\alpha}$. Set

$$
\begin{aligned}
& \mathscr{U}_{\alpha}=\left(\bigoplus_{\beta<\alpha} N_{\beta}\right) \oplus\left(\bigoplus_{\beta \geq \alpha} E_{\beta}\right) \\
& \mathscr{V}_{\alpha}=\left(\bigoplus_{\beta<\alpha} E_{\beta}\right) \oplus\left(\bigoplus_{\beta \geq \alpha} N_{\beta}\right) .
\end{aligned}
$$

Clearly the $\mathscr{U}_{\alpha}$ form a descending chain and the $\mathscr{V}_{\alpha}$ an ascending chain of order type $\kappa$. If $\mathscr{U}_{\alpha}=\mathscr{U}_{\alpha+1}$ (respectively $\mathscr{V}_{\alpha}=\mathscr{V}_{\alpha+1}$ ), taking complements modulo $\bigoplus_{\beta<\alpha} N_{\beta} \oplus \bigoplus_{\beta>\alpha} E_{\beta}$ (respectively $\bigoplus_{\beta<\alpha} E_{\beta} \oplus \bigoplus_{\beta>\alpha} N_{\beta}$ ) gives $N_{\alpha}=E_{\alpha}$ since $E_{\alpha} \supseteq N_{\alpha}$. But $N_{\alpha}$ was a proper submodule of $E_{\alpha}$, so the $\mathscr{U}_{\alpha}$ (respectively $\mathscr{V}_{\alpha}$ ) are all distinct. Thus either the ascending or descending $\aleph$-chain condition (2) implies that $|\kappa|<\aleph$.

Lemma 3. If $A \subseteq B \subseteq M$ and $L \subseteq M$, then $A=B \Longleftrightarrow A \cap L=B \cap L$ and $A+L=B+L$. In particular, if $\kappa$ is well ordered and $\left\{N_{\alpha} \mid \alpha<\kappa\right\}$ is an increasing or decreasing chain of submodules of $M$, then for any $L \subseteq M$, if $N_{\alpha}+L=N_{\beta}+L$ (respectively $N_{\alpha} \cap L=N_{\beta} \cap L$ ) for all $\beta$ with $\alpha \leq \beta<\gamma$, then $\left\{N_{\beta} \cap L \mid \alpha \leq \beta<\gamma\right\}$ (respectively $\left\{N_{\beta}+L \mid \alpha \leq \beta<\gamma\right\}$ ) is an increasing or decreasing chain of submodules of $L$ (respectively $M / L$ ).

Theorem 3. $M$ has the ascending (respectively descending) $\aleph$-chain condition 
on essential submodules $\Longleftrightarrow M / \operatorname{socle}(M)$ has the ascending (respectively descending) $\aleph$-chain condition on all submodules.

Proof. Let $S$ denote the socle of $M$. Since any essential submodule of $M$ must contain $S$, $\Longleftarrow$ follows from Lemma 3. For the converse, there is a submodule $K \subseteq M$ with $S \oplus K \subseteq^{\prime} M$. Then $M /(K \oplus S)$ has the appropriate $\aleph$-chain condition. By Lemma 3, $M / S$ has an $\aleph$-chain condition if and only if both $M /(S \oplus K)$ and $(S \oplus K) / S \cong K$ do. But $K$ has zero socle, so by Theorem 2 , every independent family of submodules of $K$ has fewer than $\aleph$ elements. Thus Theorem 1 shows that $K$ has the appropriate $\aleph$-chain condition.

Lemma 4. If $S$ is the socle of a direct sum $\bigoplus N_{\alpha}$, then $S=\bigoplus \operatorname{socle}\left(N_{\alpha}\right)$. Hence $\left(\bigoplus N_{\alpha}\right) / S \cong \bigoplus\left(N_{\alpha} / \operatorname{socle}\left(N_{\alpha}\right)\right)$

Sketch of proof. A homomorphic image of a semisimple is semisimple, so the socle of each $N_{\alpha}$ contains the projection of $S$ to $N_{\alpha}$.

Theorem 4. Let $M$ be a CS module. Then $M$ has the ascending (respectively descending) $\aleph$-chain condition on essential submodules $\Longleftrightarrow M$ is a direct sum $K \oplus L$ where $K$ has the ascending (respectively descending) $\aleph$-chain condition on all submodules and $L$ is semisimple.

Proof. $\Longleftarrow$ is clear since any essential submodule contains the socle.

For $\Longrightarrow$, by Theorem $3 M / \operatorname{socle}(M)$ has the appropriate $\aleph$-chain condition. Let $\left\{\bar{N}_{\alpha} \mid \alpha \in \kappa\right\}$ be a maximal independent family of submodules of $M / \operatorname{socle}(M)$ where each $\bar{N}_{\alpha}$ is finitely generated. Then $|\kappa|<\aleph$ and there is a family $\left\{N_{\alpha} \subseteq M \mid \alpha \in \kappa\right\}$ of finitely generated $N_{\alpha}$ such that $N_{\alpha}+\operatorname{socle}(M)=\bar{N}_{\alpha}$ for each $\alpha \in \kappa$. Let $K$ be a maximal essential extension of $\sum_{\alpha \in \kappa} N_{\alpha}$ in $M$. Then $K$ is a direct summand of $M$ since $M$ is $C S$. Let $M=K \oplus L$. By Lemma 4 , socle $(M)=\operatorname{socle}(K) \oplus \operatorname{socle}(L)$. Since $K / \operatorname{socle}(K) \subseteq^{\prime} M / \operatorname{socle}(M)=K / \operatorname{socle}(K) \oplus L / \operatorname{socle}(L), L=\operatorname{socle}(L)$ is semisimple. We next show that $\operatorname{socle}(K)$ has the ascending $\aleph$-chain condition. Since socle $(K)$ is semisimple it has a well-defined dimension as a sum of simples, so we need only show that socle $(K)$ has no independent family with $\aleph$ members. Assume socle $(K)$ does contain an independent family with $\aleph$ elements. Since $\aleph \cdot \aleph=\aleph$, $\operatorname{socle}(K)$ contains some independent family $\left\{T_{\beta}\right\}$ of cardinality $\aleph$, where each $T_{\beta}$ cannot be generated by fewer than $\aleph$ elements. For each $\beta$, let $U_{\beta}$ be a maximal essential extension of $T_{\beta}$ in $M$. By Lemma $4, \sum_{\beta} U_{\beta} / T_{\beta}$ is direct in $M / \operatorname{socle}(M)$. Thus for some $\gamma, U_{\gamma}=T_{\gamma}$, so $T_{\gamma}$ is a direct summand of $M$ and hence of any submodule containing it. $\sum_{\alpha \in \kappa} N_{\alpha}$ is a submodule of $M$ generated by fewer than $\aleph$ elements and it contains $T_{\gamma}$, so $T_{\gamma}$ is generated by fewer than $\aleph$ elements, a contradiction. Thus socle $(K)$ must be generated by fewer than $\aleph$ elements, so both $K / \operatorname{socle}(K)$ and $\operatorname{socle}(K)$ have the appropriate $\aleph$-chain condition and, by Lemma 3, so does $K$.

We remark that [4] has a weaker version of this result for $\aleph=\aleph_{0}$.

Corollary 5. Let $M$ be $C S$ and $\aleph^{\prime}$-generated for some $\aleph^{\prime}<\aleph$. Then $M$ has an $\aleph$-chain condition on essential submodules if and only if $M$ has that $\aleph$-chain condition on all submodules.

Proof. The semisimple $L$ in Theorem 4 must be $\aleph^{\prime}$-generated. It therefore has dimension $\leq \aleph^{\prime}<\aleph$ and so both $\aleph$-chain conditions hold for $L$. Apply Lemma 3.

Corollary 6. If the ring $R$ has every complement right ideal a direct summand and an $\aleph$-chain condition on essential right ideals, then $R$ has that $\aleph$-chain condition on all right ideals. 
Proof. 1 is less than any infinite cardinal.

Corollary 6 plus the Hopkins-Levitzki theorem show that a right $C S$ ring with the descending $\aleph_{0}$-chain condition on essential right ideals has finite composition length on the right.

Lemma 5. Let $M$ be a module that has acc on essential submodules. Then

$$
\mathbf{J}=\left\{\lambda \in \operatorname{End}(M) \mid \operatorname{kernel}(\lambda) \subseteq^{\prime} M\right\}
$$

is a left T-nilpotent ideal of $\operatorname{End}(M)$.

Sketch of proof. It is standard that $\mathbf{J}$ is a two-sided ideal of End $(M)$. Let $\left\{\lambda_{i} \mid i \in \omega\right\} \subseteq \mathbf{J}$. By acc, there is an $n \in \omega$ with

$$
\operatorname{kernel}\left(\lambda_{n+1} \lambda_{n} \cdots \lambda_{0}\right)=\operatorname{kernel}\left(\lambda_{n} \cdots \lambda_{0}\right) \text {. }
$$

Then kernel $\left(\lambda_{n+1}\right) \bigcap$ image $\left(\lambda_{n} \cdots \lambda_{0}\right)=0$, so by the definition of $\mathbf{J}, \lambda_{n} \cdots \lambda_{0}$ $=0$.

Corollary 7. If $M$ is a continuous module with acc on essential submodules, then $\operatorname{End}(M)$ (written on the left) is of the form

$$
\operatorname{End}(M)=\left(\begin{array}{cc}
\mathscr{S} & 0 \\
B & \Lambda
\end{array}\right)
$$

where $\mathscr{S}$ is semiprimary, $\Lambda$ is a direct product of full rings of linear transformations and $B$ is a $\Lambda-\mathscr{S}$ bimodule.

Proof. By Corollary 4, $M=K \oplus L$ where $K$ is Noetherian and $L$ is semisimple. By taking a maximal semisimple direct summand of the Noetherian module $K$ and adding it to $L$ we may assume that $K$ has no simple direct summand. Then $K$ is Noetherian and continuous, so $\mathscr{S}=\operatorname{End}(K)$ is semiperfect and $\mathbf{J}=\left\{\lambda: K \longrightarrow K \mid \operatorname{kernel}(\phi) \subseteq^{\prime} K\right\}$ is its radical. But acc on submodules of $M$ implies there is an $n \in \omega$ such that $\left(0: \mathbf{J}^{n}\right)=\left(0: \mathbf{J}^{k}\right)$ for all $k>n$ where $(0: N)$ denotes the annihilator of $N$ in $M$. Assume we have a sequence $\left\{\lambda_{0}, \lambda_{1}, \ldots, \lambda_{k}\right\}$ of elements of $\mathbf{J}$ such that $\mathbf{J}^{n} \lambda_{k} \cdots \lambda_{0} M \neq 0$. Then $\mathbf{J}^{n+1} \lambda_{k} \cdots \lambda_{0} M \neq 0$ so there is a $\lambda_{k+1} \in \mathbf{J}$ with $\mathbf{J}^{n} \lambda_{k+1} \cdots \lambda_{0} M \neq 0$. By $T$ nilpotence, this cannot happen so $\mathbf{J}^{n}=0$ (see [2, p. 324]) and $\mathscr{S}$ is semiprimary. $L$ is semisimple, so $\Lambda=\operatorname{End}(L)$ is a product of the endomorphism rings of the homogeneous components of $L$, that is, rings of the form

$$
\operatorname{Hom}\left(\bigoplus_{i \in \mathcal{I}} S_{i}, \bigoplus_{j \in \mathcal{I}} S_{j}\right) \cong \prod_{i} \bigoplus_{j} \operatorname{Hom}\left(S_{i}, S_{j}\right)
$$

which is just column finite matrices over the division ring $\operatorname{Hom}(S, S) . B$ is the $\Lambda-\mathscr{S}$ bimodule $\operatorname{Hom}(K, L) . \quad M$ is continuous and $K$ has no simple direct summands, so there can be no maps from $L$ to $K$.

If $M$ in Corollary 7 is the ring itself, we get the theorem in [6] that a continuous ring with acc on essential submodules has dcc. However, that is a very special case. There is an example of an injective module $M$ with acc but not 
dcc (see [9]). Its endomorphism ring happens to be a division ring which is most certainly semiprimary.

Appendix. Proof of Theorem 1 for NONREgular Cardinals $\aleph$

This appendix contains the second (for ascending chains) and third (for descending chains) parts of the proof of Theorem 1 . We now assume that $\aleph$ is not regular.

Theorem 1. Let $M$ be a module and $\aleph$ an infinite cardinal such that

(1) Any independent family of submodules of $M$ has fewer than $\aleph$ members.

(2) The set of all essential submodules of $M$ has the ascending (respectively descending) $\aleph$-chain condition.

Then the set of all submodules of $M$ has the ascending (respectively descending) $\aleph$-chain condition. Conversely, an $\aleph$-chain condition on all submodules of $M$ implies (1) and the same chain condition in (2).

Continuation of the proof. As in Part 1 for the regular case, we let $\left\{N_{\alpha} \mid \alpha<\kappa\right\}$ be an ascending (respectively descending) chain of distinct submodules of $M$. For all $\alpha, \beta<\kappa, \alpha \sim \beta$ iff $N_{\alpha} \subseteq^{\prime} N_{\beta}$ or $N_{\beta} \subseteq^{\prime} N_{\alpha} . \bar{\alpha}$ denotes the smallest member in its equivalence class and $\overline{\alpha^{+}}$the successor of $\bar{\alpha}$ in the set $\mathscr{C}_{\sim}=$ $\{\sim$-class representatives $\bar{\alpha}\}$.

Part 2. Ascending chains. Now assume the ascending $\aleph$-chain condition on essentials in (2), where $\aleph$ need not be regular. Since adjoining a new $N_{\lambda}$ for each limit ordinal $\lambda<\kappa$ does not increase cardinality, we may assume that the chain $\left\{N_{\alpha} \mid \alpha<\kappa\right\}$ is smooth, that is, for a limit ordinal $\lambda, N_{\lambda}$ is the union of the preceding $N_{\beta}$. As in Part 1 , let $L_{\bar{\alpha}}$ be a complement of $N_{\bar{\alpha}}$ in $N_{\overline{\alpha^{+}}}$. Let $K$ be a complement of $\bigcup_{\alpha<\kappa} N_{\alpha}$ in $M$, and set

$$
L=\left(\sum_{\mathscr{C}_{\sim}} L_{\bar{\alpha}}\right) \oplus K .
$$

We claim that $L$ is essential in $M$. Indeed, let $Z$ be a nonzero submodule of $M$. Then $Z \cap\left(K \oplus \bigcup_{\alpha<\kappa} N_{\alpha}\right) \neq 0$, so unless $\kappa=0$, using smoothness we get $Z \cap\left(K \oplus N_{\alpha}\right) \neq 0$ for some $\alpha<\kappa$ in the $\sim$-class of the $\mathscr{C}_{\sim}$-successor $\overline{\alpha_{0}{ }^{+}}$. Then by definition of $L_{\overline{\alpha_{0}}}$ we get

$$
Z \cap\left(K \oplus L_{\overline{\alpha_{0}}} \oplus N_{\overline{\alpha_{0}}}\right) \neq 0 .
$$

Again using smoothness at limit ordinals, if $\overline{\alpha_{0}} \neq 0$ there is an $\alpha<\overline{\alpha_{0}{ }^{+}}$with $\alpha$ in the $\sim$-class of a $\mathscr{C}_{\sim}$-successor $\overline{\alpha_{1}{ }^{+}}<\overline{\alpha_{0}{ }^{+}}$such that

$$
Z \cap\left(K \oplus L_{\overline{\alpha_{0}}} \oplus L_{\overline{\alpha_{1}}} \oplus N_{\overline{\alpha_{1}}}\right) \neq 0 .
$$

Continuing in this manner we get a descending sequence $\left\{\overline{\alpha_{i}}\right\} \subset \kappa$ that must terminate since $\kappa$ is well ordered. If it terminates at $\overline{\alpha_{n}}$, then

$$
Z \cap\left(K \oplus \sum_{i=0}^{n} L_{\overline{\alpha_{i}}}\right) \neq 0 .
$$

This shows that $L \subseteq^{\prime} M$. We thus get a well ordered nondecreasing chain

$$
\left\{\overline{N_{\alpha}}=L+N_{\alpha} \mid \alpha<\kappa\right\}
$$


of essential submodules of $M$. We observe that, for each $\alpha \sim \bar{\alpha}, N_{\alpha} \supseteq$ $\sum_{\bar{\beta}<\bar{\alpha}} L_{\bar{\beta}}$. Also, either $N_{\alpha} \subset^{\prime} N_{\alpha+1}$ or $\alpha+1=\overline{\alpha^{+}}$and $\left(N_{\alpha} \oplus L_{\bar{\alpha}}\right) \subseteq^{\prime} N_{\alpha+1}$. If $N_{\alpha} \subset^{\prime} N_{\alpha+1}$ then $\overline{N_{\alpha}} \neq \overline{N_{\alpha+1}}$ since $K \oplus \sum_{\bar{\gamma} \geq \bar{\alpha}} L_{\bar{\gamma}}$ is a direct sum complement of $N_{\alpha+1}$ and $N_{\alpha}$ in $\overline{N_{\alpha+1}}$ and $\overline{N_{\alpha}}$ respectively, and $N_{\alpha+1} \supset N_{\alpha}$. If $\left(N_{\alpha} \oplus L_{\bar{\alpha}}\right) \subseteq^{\prime}$ $N_{\alpha+1}$ then, by the same reasoning, $\overline{N_{\alpha+1}}=\overline{N_{\alpha}}$ if and only if $N_{\alpha+1}=\left(N_{\alpha} \oplus L_{\bar{\alpha}}\right)$.

Thus there is at most one duplication per equivalence class, and $|\kappa| \leq$ the number of distinct $\overline{N_{\alpha}}$ plus the number of equivalence classes, and both of these cardinalities are $<\aleph$.

Part 3. Descending chains.

In this portion of the proof, the $\left\{N_{\alpha}\right\}$ form a descending chain and $\aleph$ is not regular. We know that the cardinalities of $\mathscr{C}_{\sim}$ and each $\sim$-class are less than $\aleph$. Thus $|\kappa| \leq \aleph$. So now assume that $|\kappa|=\aleph$. By truncating the chain if neseccary, we may even assume that $\kappa=$ (the first ordinal with cardinality) $\aleph$.

Using transfinite induction we define a family $\left\{K_{\bar{\alpha}} \mid \bar{\alpha} \in \mathscr{C}_{\sim}\right\}$ of submodules $K_{\bar{\alpha}}$ of $M$ by setting $K_{\bar{\alpha}}$ equal to a complement of $\left(\bigoplus_{\bar{\beta}<\bar{\alpha}} K_{\bar{\beta}}\right) \oplus N_{\bar{\alpha}}$ in $M$. Set

$$
K=\bigoplus_{\bar{\alpha} \in \mathscr{C}_{\sim}} K_{\bar{\alpha}} .
$$

Clearly each $N_{\alpha}+K$ is essential in $M$. Then $\left\{N_{\alpha}+K \mid \alpha<\kappa\right\}$ has fewer than $\aleph$ distinct elements. Moreover, $\left(N_{\alpha} \cap K\right)=\left(N_{\alpha+1} \cap K\right) \Longrightarrow\left(N_{\alpha}+K\right) \neq\left(N_{\alpha+1}+K\right)$ by Lemma 3. By omitting the largest element (if it exists) of each subset of $\kappa$ on which $N_{\alpha}+K=N_{\beta}+K$, we get $\left\{K \cap N_{\alpha} \mid \alpha \in \kappa\right\}$ must have $\aleph$ distinct elements and so can be order indexed by $\kappa$. By Lemma $1, K$ has the descending $\aleph$-chain condition. Thus adapting our original notation to $K$ and $\left\{N_{\alpha} \cap K\right\}$, we may assume that

$$
M=\bigoplus_{\bar{\alpha} \in \mathscr{C}_{\sim}} K_{\bar{\alpha}} \quad \text { and } \quad N_{\bar{\alpha}} \cap\left(\bigoplus_{\bar{\beta} \leq \bar{\alpha}} K_{\bar{\beta}}\right)=0 .
$$

We will adapt the idea used in proving Theorem 2 to this situation. We find a direct sum of submodules of $M$ each summand of which contains a descending chain of submodules with an essential intersection. When the chains are inserted between intersections in preceeding summands and largest members in following summands, we get a chain of cardinality $\aleph$.

Let us introduce some terminology. A tail of $\kappa$ is a subset $\{\beta\} \subseteq \kappa$ where $\beta$ runs over the set of upper bounds of some bounded subset of $\mathscr{C}_{\sim}$. A finite subsum of $M$ is a sum $\bigoplus_{\bar{\beta} \in S} K_{\bar{\beta}}$ where $S$ is a finite subset of $\mathscr{C}_{\sim}$. We observe that any tail has cardinality $\aleph$ and there are precisely $\left|\mathscr{C}_{\sim}\right|$ finite subsums.

Now $\aleph$ is not regular, so we may find a chain $\left\{\lambda_{\sigma} \mid \sigma<\operatorname{cofinality}(\aleph)\right\}$ of cardinals with supremum $\aleph$ where for each $\sigma,\left|\mathscr{C}_{\sim}\right|<\lambda_{\sigma}<\aleph$. We will now do a transfinite induction on cofinality $(\aleph)$ using this notation.

Assume for every $\tau<\sigma$ we have a finite subset $S_{\tau}$ of $\mathscr{C}_{\sim}$ such that

(i) The finite subsum corresponding to $S_{\tau}$ contains a descending chain of order type $>\lambda_{\tau}$ consisting of submodules each of which is essential in the largest member of the chain, and

(ii) For all $v<\tau$, every element of $S_{\tau}$ is greater than every element of $S_{v}$.

We show how to find $S_{\sigma}$ preserving these properties. The set $S=\bigcup_{\tau<\sigma} S_{\tau}$ has cardinality $\leq \aleph_{0} \cdot|\sigma|<\operatorname{cofinality}(\aleph)$. Since no equivalence class has $\aleph$ 
elements, $\mathscr{C}_{\sim}$ must be cofinal in $\kappa=\aleph$. Thus $S$ is not cofinal in $\mathscr{C}_{\sim}$. The tail corresponding to $S$ has cardinality $\aleph$, so there is some $\overline{\alpha_{\sigma}}$ in this tail with $\left|\left\{\beta \sim \overline{\alpha_{\sigma}}\right\}\right|>\lambda_{\sigma}$. For $\beta \sim \overline{\alpha_{\sigma}}$, let $\bar{N}_{\beta}$ denote the projection of $N_{\beta}$ to $\bigoplus_{\bar{\gamma}>\overline{\alpha_{\sigma}}} K_{\bar{\gamma}}$. Note that this projection is one-to-one on $N_{\overline{\alpha_{\sigma}}}$ and so preserves the distinctness of the $N_{\beta}$ for $\beta \sim \overline{\alpha_{\sigma}}$.

For every $\beta \sim \overline{\alpha_{\sigma}}$, let $H_{\beta}$ be a finitely generated submodule of $\bar{N}_{\beta}$ with $H_{\beta}$ not contained in $\bar{N}_{\beta+1}$. This is the categorical equivalent of taking an $x \in$ $\bar{N}_{\beta} \backslash \bar{N}_{\beta+1}$. By exact direct limits, each $H_{\beta}$ is in a finite subsum corresponding to a finite subset of $\left\{\bar{\gamma} \in \mathscr{C}_{\sim} \mid \bar{\gamma}>\overline{\alpha_{\sigma}}\right\}$. There are at most $\left|\mathscr{C}_{\sim}\right|$ such finite subsums and more than $\lambda_{\sigma}>\left|\mathscr{C}_{\sim}\right|$ of the $H_{\beta}$, so not every finite subsum can contain $\leq \lambda_{\sigma}$ of the $H_{\beta}$. We thus have a finite set $S_{\sigma}$ of elements of $\mathscr{C}_{\sim}$ which are all greater than $\overline{\alpha_{\sigma}}$ and thus upper bounds of $\bigcup_{\tau<\sigma} S_{\tau}$ with the property that the finite subsum $L_{\sigma}=\bigoplus_{\bar{\beta} \in S_{\sigma}} K_{\bar{\beta}}$ contains more than $\lambda_{\sigma}$ of the $H_{\beta}$. Since $\beta \sim \overline{\alpha_{\sigma}},\left(\bar{N}_{\beta} \cap L_{\sigma}\right) \subseteq^{\prime}\left(\bar{N}_{\overline{\alpha_{\sigma}}} \cap L_{\sigma}\right)$. But $H_{\beta} \subseteq L_{\sigma} \Longrightarrow \bar{N}_{\beta} \cap L_{\sigma}$ properly contains $\bar{N}_{\beta+1} \cap L_{\sigma}$, so $S_{\sigma}$ satisfies (i) and (ii).

Let $H_{\sigma}$ denote a complement of $\bar{N}_{\overline{\alpha_{\sigma}}} \cap L_{\sigma}$ in $L_{\sigma}$ and set

$$
H=\bigoplus_{\sigma<\text { cofinality }(\aleph)} H_{\sigma} .
$$

Let $B_{\sigma}$ be the intersection of the first $\lambda_{\sigma}$ distinct $\bar{N}_{\beta} \cap L_{\sigma}$ with $\beta \sim \overline{\alpha_{\sigma}}$. Then $H_{\sigma} \oplus B_{\sigma} \subseteq^{\prime} L_{\sigma}$.

Then the set

$$
\begin{array}{r}
\left\{H \oplus \sum_{\tau<\sigma} B_{\tau} \oplus\left(B_{\sigma}+\bar{N}_{\beta} \cap L_{\sigma}\right) \oplus \sum_{\tau>\sigma}\left(\bar{N}_{\bar{\alpha}_{\tau}} \cap L_{\tau}\right) \mid\right. \\
\left.\sigma<\operatorname{cofinality}(\aleph), \beta \sim \overline{\alpha_{\sigma}}\right\}
\end{array}
$$

is a nonascending chain of essential submodules of $\bigoplus_{\sigma<\operatorname{cofinality}(\aleph)} L_{\sigma}$ which, after duplications are eliminated, can be indexed by an ordinal with cardinality $\geq$ every $\lambda_{\sigma}$. Such an ordinal has cardinality at least $\aleph$, so this gives us our long awaited contradiction.

\section{REFERENCES}

1. E. P. Amendariz, Rings with dcc on essential left ideals, Comm. Algebra 8 (1980), 299-308.

2. F. W. Anderson and K. R. Fuller, Rings and categories of modules, Graduate Texts in Math., No. 13, Springer-Verlag, New York, Heidelberg, and Berlin, 1974.

3. H. Bass, Descending chains and the Krull ordinal of commutative Noetherian rings, J. Pure Appl. Algebra 1 (1971), 347-360.

4. V. Camillo and M. F. Yousif, CS-modules with acc or dcc on essential submodules, preprint.

5. N. V. Dung, D. V. Huynh, and R. Wisbauer, Quasi-injective modules with acc or dcc on essential submodules, Arch. Math. (to appear).

6. S. K. Jain, S. R. López-Permouth, and S. T. Rizvi, Continuous rings with acc on essentials are Artinian, Proc. Amer. Math. Soc. (to appear).

7. A. V. Jategaonkar, A counterexample in ring theory and homological algebra, J. Algebra 12 (1969), 418-440.

8. J. Lawrence, A countable self-injective ring is quasi-Frobenius, Proc. Amer. Math. Soc. 665 (1977), 217-220. 
9. R. W. Miller and D. R. Turnidge, Some examples from infinite matrix rings, Proc. Amer. Math. Soc. 38 (1973), 65-67.

10. B. L. Osofsky, Projective dimension of "nice" directed unions, J. Pure Appl. Algebra 13 (1978), 179-219.

Department of Mathematics, Rutgers University, New Brunswick, New Jersey 08903

E-mail address: osofsky@math.rutgers.edu 